\title{
Posterior Capsulotomy Rate And Dysphotopsias Following Implantation Of Hoya iSert 250 Monofocal Intraocular Lens: 24 Month Study
}

\author{
Ejaz Ansari
}

\begin{abstract}
Purpose: to evaluate dysphotopsias, patient satisfaction and posterior capsulotomy rate in patients undergoing phacoemulsification and implantation with Hoya iSert 250 monofocal intraocular lens (IOL).

Design: retrospective cohort study. Setting- Teaching unit at a District General Hospital, UK.

Methods: patients were implanted with the hydrophobic acrylic Hoya iSert 250 monofocal IOL through a $2.2 \mathrm{~mm}$ clear corneal/ limbal incision. For dysphotopsia data, patients responded to a telephone questionnaire describing any visual symptoms and their satisfaction. Nd:YAG laser capsulotomy data were collected retrospectively from electronic medical records, paper notes and laser records. Eyes from patients (mean age 73.6 range 64-93 years) undergoing cataract surgery with implantation of Hoya iSert 250 IOL during the period January 1, 2014 and July 31, 2014, and 2-year follow-up were analysed.
\end{abstract}

Main outcome measures: dysphotopsia rate, patient satisfaction and posterior capsulotomy rate.

Results: 106 patients had the Hoya iSert 250 lenses implanted over a seven month period in 2014 . 88 patients were contacted at 24 months post- operatively. 18 had passed away or were not contactable. 61 patients completed the survey. 15 patients $(24.5 \%)$ reported transient positive dysphotopsias. One patient $(1.6 \%)$ reported negative dysphotopsia. $95 \%$ were either very satisfied or satisfied with their vision $(59 \%$ 'very satisfied' and $36 \%$ 'satisfied'). 3 patients $(4.9 \%)$ had a YAG laser capsulotomy within 24 months post-operatively.

Conclusions: the Hoya iSert 250 monofocal IOL was associated with low dysphotopsia and posterior capsulotomy rates with the majority of patients being 'Very Satisfied' with their vision at 24 months.

Posterior capsulotomy rate and dysphotopsias following implantation of Hoya iSert 250 monofocal intraocular lens: 24 month study.

Index Terms - Capsulotomy; Nd: YAG; dysphotopsia; hydrophilic; hydrophobic.

\section{INTRODUCTION}

Modern cataract surgery has a very high success rate with studies quoting corrected distance visual acuity (CDVA) of at least 0.5 (6/12 Snellen) being achieved in $84 \%$ to $98.5 \%$ of cases [1]-[3]. Despite this success rate there are potential complications, the most common being posterior capsule opacification (PCO). PCO can develop in a few months to a few years after surgery with incidence ranging between 5$50 \%$ [4]. Its development is determined by various factors

Published on June 17, 2020.

E. Ansari, Maidstone and Tunbridge Wells NHS Trust, UK.

(corresponding e-mail: e.ansari@nhs.net). including intraocular lens (IOL) material, IOL edge design and haptic design [5].

Neodymium-doped yttrium aluminium garnet (Nd:YAG) laser capsulotomy ("YAG capsulotomy") is a very safe procedure which is performed routinely to treat PCO. In most cases its effects in improving vision are almost immediate with very few complications reported [6]-[8]. However, this places an enormous burden on health services both financially and in terms of capacity and time because of the huge and increasing numbers of cataract procedures that are performed annually [9], which translates into a large number of YAG capsulotomy procedures. In addition, PCO is a disappointment and inconvenience to patients.

IOLs which are associated with lower rates of PCO and YAG capsulotomy are naturally the preferred option to help reduce this demand on health services. Studies have shown that hydrophobic acrylic IOLs are associated with a longer period of time until the need for YAG capsulotomy and less frequent and less dense PCO [10]-[12].

Dysphotopsias are optical disturbances that represent another group of side effects of implant surgery, which can be particularly troublesome for patients. Positive dysphotopsias include haloes, glare, fog, photophobia and rainbows. Negative dysphotopsias are described as temporal shadows or crescents that are usually temporary. Dysphotopsias are a key reason for patient disappointment and can lead to IOL explantation for some [13], [14].

The HOYA iSert 250 intraocular lens is a clear, UVprotecting hydrophobic acrylic IOL that is fully preloaded. The Aspheric Balanced Curve (ABC) design provides a sharp visual image while the square edge of the lens helps to minimize posterior capsule opacification. However, hydrophobic acrylic IOLs have been shown to be associated with a higher rate of dysphotopsias than hydrophilic IOLs [15]. Therefore, we wanted to study both the rates of YAG capsulotomy and dysphotopsias following implantation of this IOL.

\section{METHOdS AND PATIENTS}

This was a single-centre cohort study including 106 patients (average age, 73.6 years (range, 64-93 years)) with no ocular co-morbidity, who had phacoemulsification surgery at Maidstone \& Tunbridge Wells Hospitals, UK by one surgeon (EA). The inclusion criteria were eyes that underwent cataract surgery between January 1, 2014 and July 31, 2014, with placement of monofocal Hoya iSert 250 IOLs into the capsular bag. Results from one eye of each patient were included. 
Exclusion criteria included a record of co-surgeries or complications that could influence the development of PCO, for example the use of capsular tension rings, anterior vitrectomy and posterior capsule (PC) rupture. All patients provided written informed consent prior to study procedures. The study conformed to the tenets of the Declaration of Helsinki. The Local Research and Development Committee had ruled that ethics approval was not required for this study.

All patient data was fully anonymised and compliant with the Data Protection Act of 1998. All follow-up data including cases of $\mathrm{Nd}$ :YAG capsulotomy were extracted from the Trust's archived electronic medical records ("enotes") or paper records if e-notes were unavailable. Laser record books were also checked at three hospitals covering the entire geographical area. This cross-checking ensured that no cases were missed.

At the two-year post-operative date, all patients completed a questionnaire similar to earlier published studies [16], [17] (Table I), describing any ocular symptoms and their satisfaction with the procedure.

\section{TABLE I: STUDY QUESTIONNAIRE}

\begin{tabular}{l} 
Did you notice any of the following symptoms following your \\
cataract operation? \\
\hline $1 . \quad$ Light sensitivity, haloes, arcs, flashes of light \\
Are these minimal/ annoying/ unbearable \\
Have they persisted since your operation? \\
\hline 2. Glare (dazzle) symptoms \\
Are these minimal/ annoying/ unbearable \\
Have they persisted since your operation? \\
\hline 3. Shadows at the side of your vision \\
Are these minimal/ annoying/ unbearable \\
Have they persisted since your operation? \\
\hline \hline How pleased are you with the results of the operation? \\
Completely satisfied \\
Satisfied \\
Neutral \\
Dissatisfied \\
Completely dissatisfied
\end{tabular}

For statistical analysis, STATA version 15.1 was used. In univariate analysis, for a continuous variable, independent sample t-test was used and a significance level of $p<0.05$ was chosen.

\section{A. Surgical procedure}

All cases were performed under topical anaesthesia. A 2.2 $\mathrm{mm}$ corneal incision was fashioned and a circular central capsulorrhexis was completed to allow complete overlap of the edge of the IOL optic. After removing each nuclear quadrant by phacoemulsification, viscoelastic (Amvisc, Bausch and Lomb, Kingston-upon-Thames, UK) was used to expand the capsular bag, and the Hoya iSert 250 IOL was implanted into the bag without enlarging the $2.2 \mathrm{~mm}$ incision. Viscoelastic was removed and none of the corneal incisions were sutured. All cases were routine and postoperative follow-up was at 3 weeks and 3 months.

\section{RESUlTS}

Post-operative vision and refraction data were available for 106 eyes (Table II). 88 (83\%) patients were contacted at 24 months post-operatively. 18 (17\%) had passed away or were not contactable.

$61(69 \%)$ patients completed the questionnaire. 15 patients $(24.5 \%)$ reported transient positive dysphotopsia (glare, light sensitivity, haloes, arcs and flashes of light) that lasted no more than 1 month. One patient (1.6\%) reported negative dysphotopsia (temporal crescent effect). 95\% were either very satisfied or satisfied with their vision (59\% 'very satisfied' and $36 \%$ 'satisfied').

3 patients $(4.9 \%)$ had YAG laser capsulotomy by 24 months post-operatively. None of the patients had unbearable symptoms and at the time of the questionnaire survey, none had persistent symptoms.

\begin{tabular}{lcc}
\multicolumn{3}{c}{ TABLE II: BASELINE CHARACTERISTICS AND VISUAL ACUITIES } \\
\hline Age (years) & 73.6 & $\begin{array}{c}\text { Range } \\
64-93\end{array}$ \\
\hline Gender & F:M 1.5:1 & $\begin{array}{c}\text { All } \\
\text { Caucasian }\end{array}$ \\
\hline $\begin{array}{l}\text { Mean pre-operative } \\
\text { corrected acuity } \\
\text { (decimalised }\end{array}$ & 0.55 & $\begin{array}{c}\text { Range } \\
\text { Snellen) }\end{array}$ \\
\hline $\begin{array}{l}\text { Mean post-operative } \\
\text { corrected acuity } \\
\text { (decimalised }\end{array}$ & 1.03 & $0.1-0.9$ \\
Snellen) & $\mathrm{P}<0.05$ & $\begin{array}{c}\text { Range } \\
\text { Mean post-operative }\end{array}$ \\
\hline \begin{tabular}{l} 
astigmatism \\
\hline
\end{tabular} & -0.39 & \\
\hline
\end{tabular}

\section{DISCUSSION}

We believe that this is the first study describing clinical performance of the Hoya iSert 250 monofocal intraocular lens (IOL). This is a clear UV-protecting hydrophobic acrylic single-piece C-loop IOL with a 360 degree square edge designed to reduce PCO formation. The overall diameter is $12.5 \mathrm{~mm}$ with a $6.0 \mathrm{~mm}$ optic that has high refractive index and is an ultraviolet- absorbing (transmission is $>95 \%$ in the range of visible light) acrylic copolymer of phenylethylmethacrylate (refractive index is 1.520 at 23 degrees Celcius). It has blue-tinted polymethylmethacrylate (PMMA) at the distal ends of the haptics and is delivered pre-loaded via a screw-down motion inserter (figure 1). The Hoya iSert 251 is a yellow blueblocking IOL with the same design (information obtained from Hoya Surgical Optics site: https://hoyasurgicaloptics.com).

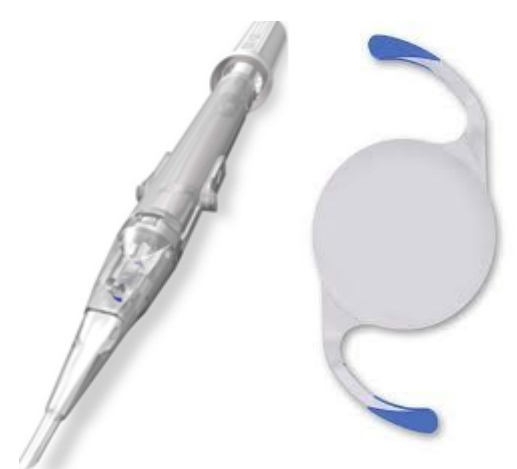

Fig. 1. Hoya iSert 250 intraocular lens and inserter. 
The IOL has a proven Aspheric Balanced Curve design that provides patients with sharp images. ABC design is an optimized surface by HOYA Lathe-Cut technology for compensation of the corneal aberrations with an asphericity of $\sim-0.18 \mu \mathrm{m}$. This allows the HOYA ABC design to provide sharp image and contrast quality even in the case of pupil offset to the capsular bag, and in most cases the eyes are left with no residual asphericity [18].

In the current study the positive and negative dysphotopsia rates were similar to previous studies of hydrophobic acrylic IOLs [16.] Hydrophobic IOLs are associated with a higher rate of dysphotopsias than hydrophilic IOLs, but in this and previous studies, the symptoms were short-lived and never debilitating.

The relationship between pseudophakic dysphotopsia and IOL design have been discussed in the literature. In one study, unwanted optic phenomena were more frequently associated with a square-edged IOL than with an IOL having a round anterior and posterior square edge [19].

In an 8 weeks randomised prospective study of 61 cases, the rate of positive and negative dysphotopsia was higher following implantation with the SN60-AT® IOL (Alcon, Fort Worth, Texas) compared to implantation with the hydrophilic Akreos Adapt ${ }^{\circledR}$ IOL (Bausch \& Lomb, Rochester, New York, USA) (31.3\% versus $20.7 \%$ ). These IOLs have similar features being square-edged, single piece with $6 \mathrm{~mm}$ optic, however the lower radius of curvature and the lower refractive index of the Akreos Adapt IOL could explain the difference in dysphotopsia rate compared to the SN60-AT® IOL [20].

In another comparative study (AcrySof ${ }^{\circledR}$ MA30BA and MA60BM [Alcon] 3-piece IOLs versus Akreos Fit巴 singlepiece IOL) [21] a lower rate of dysphotopsia was noted with the Akreos IOL when compared to the Acrysof IOLs. It was suggested that other aspects of IOL design apart from edge characteristics could explain this difference. For example,

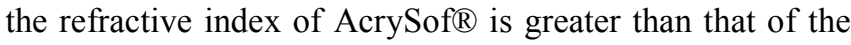
Akreos (1.56 v 1.46), which causes more surface reflections and, therefore, more dysphotopsia.

The increased incidence of post-operative dysphotopsia associated with acrylic lenses with flattened edges was verified in Tester et al study comparing AcrySof ${ }^{\circledR}$ MA30 and MA60 with Silicone SI-40 IOLs and phakic controls 16. A non-comparative study of a single-piece hydrophilic acrylic IOL, the C-flex ${ }^{\circledR}$ IOL (Rayner Intraocular Lenses Limited, Hove, UK) demonstrated that although the rate of glare was similar to other types of IOL reported in Tester's study, the incidence of unwanted imagery was significantly less [15].

In the current study, 15 patients $(24.5 \%)$ reported transient positive dysphotopsia (glare, light sensitivity, haloes, arcs and flashes of light). This is a total figure of all the symptoms listed in parentheses. In other studies, e.g. by Tester et al, the rates of individual symptoms were reported separately, but when added together, the overall rate was greater than in the current study. Therefore, the incidence of dysphotopsia associated with the Hoya iSert 250 IOL in our study compares positively with that of other IOLs reported in the literature.

The term negative dysphotopsia describes a scotoma in the temporal visual field, which is an unwanted optical sensation that can occur after uncomplicated modern cataract surgery. The incidence can be up to $15 \%$ immediately after surgery reducing to $3 \%$ after one year [22]. Negative dysphotopsia can occur with any type of IOL placed within the capsular bag. One of the major factors associated with this phenomenon is when the anterior capsule overlaps the anterior IOL edge [23], [24]. Procedures used to treat these symptoms include nasal anterior capsulotomy [25], [26] and reverse optic capture [24]. IOLs with innovative designs could prevent negative dysphotopsia [24]. In the current study there was only one case $(1.6 \%)$ of negative dysphotopsia, which resolved within 3 months.

Although hydrophilic acrylic IOLs generally lead to fewer dysphotopsias than hydrophobic IOLs, hydrophobic IOLs are concomitant with a lower rate of PCO and $\mathrm{Nd}$ :YAG laser capsulotomy. A five year study from Sweden found that the Nd:YAG laser capsulotomy rate in eyes implanted with the hydrophilic IOL was double that of the sharp-edged hydrophobic IOL group [27]. Another large Swedish study found that at 3.5 years the Nd:YAG laser capsulotomy rate was higher in the hydrophilic IOL implanted eyes [28].

A recent large longitudinal real world study from the UK confirmed these findings at 3 years, with the analysis presenting a clear advantage of hydrophobic IOLs in reducing Nd:YAG capsulotomy rates, with Acrysof IOLs performing the best [29]. This finding was repeated in a subsequent 5 year analysis by the same group [30].

Apart from IOL material and square edge characteristics, other aspects of IOL design have been studied. For example, higher Nd:YAG capsulotomy rates were found with singlepiece acrylic IOLs compared to 3-piece IOLs [31], [32]. However, this was not supported in Ursell's study that showed a reduced rate of PCO and Nd:YAG capsulotomy in the one-piece Acrysof IOL group [29].

There is also disagreement in the literature regarding square-edge profile of IOLs and Nd:YAG capsulotomy rates. For example, a paired-eye study comparing implantation of a continuous optic edge hydrophobic (not AcrySof) IOL in one eye with an AcrySof IOL having an interrupted optic edge implanted in the other eye, concluded that there was no statistically significant difference in PCO rate between the two IOL types [33]. Another study actually found a statistically significant reduction in PCO rate in cases implanted with an IOL having a continuous $360^{\circ}$ square edge [34]. Ursell's findings were again different AcrySof IOLs with an interrupted edge and one- piece design had a lower incidence of PCO and significantly lower rate of Nd:YAG capsulotomy compared to one-piece acrylic hydrophilic and hydrophobic IOLs with a continuous edge [29]. The discrepancies between studies could be explained by the differences in sample sizes and duration of follow-up.

In the current study, implantation with the Hoya iSert 250 monofocal IOL was concomitant with a Nd:YAG laser capsulotomy rate of $4.9 \%$ at 2 years, which was similar to the non- Acrysof hydrophobic IOL group in Ursell's studies. In the current study, ocular co-morbidity (age related macular degeneration, diabetic retinopathy, glaucoma), periand post-operative complications were excluded. Therefore 
the Nd:YAG capsulotomy rate in this study is free from these potential confounders.

Nd:YAG capsulotomy cases were easily accessible from the electronic and paper notes with cross-checking of laser record registers at three main hospitals in our region. This process reduced the chances of cases being missed, but like any other record systems there may have been case notes that were incomplete. The questionnaire relating to the dysphotopsia data was completed by $69 \%$ of the patients who were contacted. Therefore, the dysphotopsia rate could have been higher than what was reported in this study.

In conclusion, the Hoya iSert 250 single-piece monofocal hydrophobic IOL, which has a $360^{\circ}$ enhanced square edge, was associated with a high level of patient satisfaction and postoperative visual results in this study demographic. There was a low incidence of dysphotopsia, with symptoms being transient and not troublesome. The low Nd:YAG capsulotomy rate was comparable to IOLs of similar design and material. Overall, this translates into a better visual experience for patients in this age group and potentially fewer Nd:YAG capsulotomy procedures, thereby reducing the demand on limited healthcare services.

\section{VALUE STATEMENT}

\section{A. What Was Known}

1. Hydrophobic acrylic IOLs with a sharp $360^{\circ}$ square edge are associated with a lower PCO and Nd:YAG laser capsulotomy rate than hydrophilic acrylic IOLs.

2. However, hydrophobic acrylic IOLs with a sharp square edge are associated with a higher dysphotopsia rate than hydrophilic IOLs.

3. Prior to this study there was no data available on clinical performance of the Hoya iSert 250 monofocal 1-piece IOL, which has a hydrophobic acrylic optic and a $360^{\circ}$ square edge.

\section{B. What This Paper Adds}

1. This is the first study on clinical performance of the Hoya iSert 250 monofocal IOL.

2. Dysphotopsias with this IOL were transient, lasting less than 3 months, and the dysphotopsia rate was less than that other IOLs of the same material and design.

3. Nd:YAG laser capsulotomy rate was similar to IOLs of the same material and design.

4. The Hoya iSert 250 monofocal IOL was very userfriendly and safe, being pre-loaded, and patient satisfaction was very high.

\section{REFERENCES}

[1] Lundstrom M, Stenevi U, Thorburn W. The Swedish National Cataract Register: a 9-year review. Acta Ophthalmol Scand 2002; 80:248-257.

[2] Lundstrom M, Barry P, Leite H, Seward U, Stenevi U. 1998 European Cataract Outcome Study; report from the European Cataract Outcome Study Group. J Cataract Refract Surg 2001; 27:1176-1184.

[3] Hahn U, Krummenauer F, Kolbl B, Neuhann T, Schayan- Araghi K, Schmickler S, von Wolff K, Weindler J, Will T, Neuhann I. Determination of valid benchmarks for outcome indicators in cataract surgery; a multicenter, prospective cohort trial. Ophthalmology 2011; 118:2105-2112.

[4] Raj SM, Vasavada AR, Johar SR, Vasavada VA, Vasavada VA. Postoperative capsular opacification: a review. Int J Biomed Sci. 2007; 3:237-50.
[5] NICE. Cataracts in adults: management. Full guideline. United Kingdom: NICE; 2017.

[6] American Academy of Ophthalmology. Nd:YAG laser posterior capsulotomy. https://www.aao.org/munnerlyn-laser-surgery-center/ ndyag-laser-posterior-capsulotomy-3.

[7] Perez-Vives C. Biomaterial influence on intraocular lens performance: an overview. J Ophthalmol. 2018;2018: 2687385.

[8] Kossack N, Schindler C, Weinhold I, Hickstein L, Lehne M, Walker $\mathrm{J}$, et al. German claims data analysis to assess impact of different intraocular lenses on posterior capsule opacification and related healthcare costs. J Public Health. 2018;26: 81-90.

[9] The Way Forward- Cataract. https://www.rcophth.ac.uk/wpcontent/uploads/2015/10/RCOphth-The-Way-Forward-Cataract300117.pdf.

[10] Auffarth GU, Brezin A, Caporossi A, Lafuma A, Mendicute J, Berdeaux G. Comparison of Nd:YAG capsulotomy rates following phacoemulsification with implantation of PMMA, silicone, or acrylic intra-ocular lenses in four European countries. Ophthalmic Epidemiol. 2004; 11:319-29.

[11] Ernest PH. Posterior capsule opacification and neodymium: YAG capsulotomy rates with AcrySof acrylic and PhacoFlex II silicone intraocular lenses. J Cataract Refract Surg. 2003; 29:1546-50.

[12] Chang A, Kugelberg M. Posterior capsule opacification 9 years after phacoemulsification with a hydrophobic and a hydrophilic intraocular lens. Eur J Ophthalmol. 2017;27: 164-8.

[13] Mamalis N (2000) Complications of foldable intraocular lenses requiring explantation or secondary intervention- 1998 survey. J Cataract Refract Surg 26: 810-816.

[14] Kinnard K, Jarstad A, Olson RJ (2013) Correlation of visual quality with satisfaction and function in a normal cohort of pseudophakic patients. J Cataract Refract Surg 39: 590-597.

[15] Vyas, J. Incidence of Dysphotopsia in Patients Implanted with the Cflex ${ }^{\circledR}$ Intraocular Lens with $360^{\circ}$ Enhanced Edge: A QuestionnaireBased Study. Clin Exp Ophthalmol 2015, 6:1

[16] Tester R, Pace NL, Samore M, Olson RJ (2000) Dysphotopsia in phakic and pseudophakic patients: incidence and relation to intraocular lens type. J Cataract Refract Surg 26: 810-816.

[17] Wallin TR, Hinckley M, Nilson C, Olson RJ. A clinical comparison of single piece and three-piece truncated hydrophobic acrylic intraocular lenses. Am J Ophthalmol 2003; 136: 614-619.

[18] Mester U, Heinen S, Kaymak H. Clinical results of the aspheric intraocular lens FY-60AD (Hoya) with particular respect to decentration and tilt. Ophthalmologe. 2010 Sep;107(9):831-6. [Article in German].

[19] Bournas P, Drazinos S, Kanellas D, Arvanitis M, Vaikoussis E Dysphotopsia after cataract surgery: comparison of four different intraocular lenses. Ophthalmologica. 2007;221(6):378-83.

[20] Radford SW, Carlsson AM, Barrett GD. Comparison of pseudophakic dysphotopsia with Akreos Adapt and SN60-AT intraocular lenses. J Cataract Refract Surg. 2007 Jan;33(1):88-93.

[21] Shambhu S, Shanmuganathan VA, Charles SJ. The effect of lens design on dysphotopsia in different acrylic IOLs. Eye (Lond). 2005 May;19(5):567-70.

[22] Osher RH. Negative dysphotopsia: long-term study and possible explanation for transient symptoms. J Cataract Refract Surg 2008; 34:1699- 1707.

[23] Holladay JT, Simpson MJ. Negative dysphotopsia: causes and rationale for prevention and treatment. J Cataract Refract Surg 2017; 43:263-275.

[24] Masket S, Fram N, Cho A, et al. Surgical management of negative dysphotopsia. J Cataract Refract Surg 2018; 44:6-16.

[25] Folden DV. Neodymium: YAG laser anterior capsulectomy: surgical option in the management of negative dysphotopsia. J Cataract Refract Surg 2013; 39:1110-1115.

[26] Cooke DL, Kasko S, Platt LO. Resolution of negative dysphotopsia after laser anterior capsulotomy. J Cataract Refract Surg 2013; 39:1107-1109.

[27] Johansson B. Clinical consequences of acrylic intraocular lens material and design: Nd:YAG-laser capsulotomy rates in 3 x 300 eyes 5 years after phacoemulsification. Br J Ophthalmol. 2010;94:450-5.

[28] Cullin F, Busch T, Lundström M. Economic considerations related to choice of intraocular lens (IOL) and posterior capsule opacification frequency - A comparison of three different IOLs. Acta Ophthalmol. 2014;92:179-83.

[29] Ursell PG, Dhariwal M, Majirska K, Ender F, Kalson-Ray S, Venerus A, et al. Three-year incidence of Nd:YAG capsulotomy and posterior capsule opacification and its relationship to monofocal acrylic IOL biomaterial: a UK real world evidence study. Eye. 2018;32:1579-89.

[30] Ursell PG, Dhariwal M, O'Boyle D, Khan J, Venerus A. 5 year incidence of YAG capsulotomy and PCO after cataract surgery with 
single-piece monofocal intraocular lenses: a real-world evidence study of 20,763 eyes. Eye (2019) Eye https://doi.org/10.1038/s41433019-0630-9.

[31] Chang DF. Single versus three piece acrylic IOLs. Br J Ophthalmol. 2004;88:727-8.

[32] Mylonas G, Prskavec M, Baradaran-Dilmaghani Ret al. Effect of a single-piece and a three-piece acrylic sharp-edged IOL on posterior capsule opacification. Curr Eye Res. 2013;38:86-90.

[33] Kahraman G, Amon M, Ferdinaro C, Nigl K, Walch M. Intraindividual comparative analysis of capsule opacification after implantation of 2 single-piece hydrophobic acrylic intraocular lenses models: three-year follow-up. J Cataract Refract Surg. 2015;41:9906.

[34] Nixon DR, Woodcock MG. Pattern of posterior capsule opacification models 2 years postoperatively with 2 single-piece acrylic intraocular lenses. J Cataract Refract Surg. 2010;36:929-34.

Professor Ejaz Ansari is a Consultant, Ophthalmic Surgeon at Maidstone \& Tunbridge Wells Hospitals with an academic affiliation at The University of Kent, UK.

He obtained his BSc(Hons) (1987), medical degree MBBCh (1990) and postgraduate doctorate MD (2003) at the University of Wales. He became Fellow of the Royal College of Ophthalmologists, London 1995.

His main research interests are in cataract surgery and glaucoma, in which he has published extensively.

Professor Ansari is a member of the European Glaucoma Society and European Society for Cataract and Refractive Surgery. 